\title{
Characterization of Superplastic Behavior of AA2195 by Bulging Test
}

\author{
Ho-Sung Lee ${ }^{1, a *}$ Jong-Hoon Yoon ${ }^{1, b}$, Joon-Tae Yoo ${ }^{1, c}$, and Kyung-Ju Min ${ }^{2, d}$ \\ ${ }^{1}$ Launcher Structure \& Materials Team, Korea Aerospace Research Institute, \\ 169-84 Gwahangno, Yuseong-Gu, Daejeon 34133 Republic of Korea \\ ${ }^{2}$ Aerospace Application \& Materials Research Team, Korea Aerospace Research Institute, \\ 169-84 Gwahangno, Yuseong-Gu, Daejeon 34133 Republic of Korea

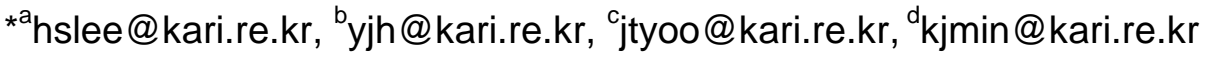

Keywords: Superplasticity, AA2195, Bulging test, Mechanical properties, Elevated temperature

\begin{abstract}
Superplasticity is the ability of polycrystalline materials to deform plastically to exhibit very large strains. Using this property in manufacturing process makes many of aerospace components lighter and stiffer, with efficient energy and cost saving. Since in most sheet metal forming operation biaxial as well as uniaxial stress state exists, it is important to understand superplastic behavior as a function of stress state. The main requirement of superplastic deformation includes high strain rate sensitivity, lack of necking, limited range of strain rate, and elevated temperatures. In this study, materials properties of 2195 aluminum-lithium alloy during superplastic deformation were obtained from biaxial bulging test. Aluminum-lithium alloy is a light weight metal that has been used as substitute for conventional aerospace aluminum alloys. At constant gas pressure, the strain rate in which the metal sheet experiences varies and therefore the strain rate sensitivity can be obtained in a single loading. Tensile tests were performed at elevated temperature and materials parameters were compared with the one obtained from biaxial bulging tests.
\end{abstract}

\section{Introduction}

Most commercially important superplastic materials exhibit "micrograin superplasticity". This superplasticity involves microstructural stability of materials during deformation. A stable, fine and equiaxed grain size is essential at elevated temperature superplasticity. The microstructural requirements for superplasticity [1] are (a) a fine and equiaxed grain size, usually less than 10 microns, (b) multiple phases or fine precipitates to suppress grain growth and (c) high angle grain boundaries which are mobile. In order to maintain this stable microstructure during elevated temperature deformation, multiphase alloys like eutectic or eutectoid alloys with equal portions of both phases and quasi-single phase alloy with fine precipitates to inhibit grain growth during static or dynamic recrystallization have been known as adequate superplastic alloys. The former examples are $\mathrm{Pb}-\mathrm{Sn}$ and Bi-Sn eutectics, Zn-22\% Al eutectoid, duplex steels, and two phase titanium alloys, and the latter, aluminum alloys. Since superplasticity is diffusion controlled process, the deformation temperature is usually above half the melting temperature in absolute temperature scale. At these elevated temperatures, dynamic recovery and dynamic recrystallization becomes active and flow stress and hardening rate decrease with increasing temperature. Increasing deformation temperature has a similar effect as decreasing grain size. It is assumed that there is equilibrium between recovery and hardening at these temperatures so that the metal does not strain harden. This is important implications in practical application because the metal will have similar microstructure and mechanical properties before and after deformation.

At elevated temperature deformation, the thermally activated component of the flow stress has been represented in a form of the following equation (Mukherjee-Bird-Dorn equation) [2]:

$$
\frac{\dot{\varepsilon} k T}{G b D}=A\left[\frac{\sigma}{G}\right]^{n}\left[\frac{b}{d}\right]^{p}
$$


where, $\sigma$ is the flow stress, $G$ the shear modulus, $b$ the Burgers vector, $d$ the grain size, $n$ the stress exponent, $\mathrm{p}$ the strain rate dependence on grain size, $\mathrm{k}$ the Boltzman constant, $\dot{\varepsilon}$ the strain rate, $\mathrm{D}$ the appropriate diffusivity, $\mathrm{T}$ the absolute temperature, and $\mathrm{A}$ is a microstructure and mechanism dependent constant which varies with the controlling deformation mechanism [3]. It is important to understand superplasticity in the development of innovative superplastic forming and manufacturing processes. In superplastic forming process of sheet metals, the actual stress state is biaxial which is different from the uniaxial state. Therefore, a biaxial bulging test provides more practical information for industrial superplastic forming practice. A2195 is the third generation Al-Li alloy, which has been successfully used in Space Shuttle external fuel tank [4]. This is because Li enhances the elastic modulus of the alloy, nearly $6 \%$ per unit weight $\%$ of $\mathrm{Li}$ addition [5]. The present work is to characterize the superplastic behavior of Al-Li 2195 alloy for superplastic forming of space launcher part. By performing biaxial bulging test with constant gas pressure, the optimum superplastic forming condition were obtained with materials parameters to predict the forming behavior. The result was compared with that from elevated temperature tensile tests.

\section{Experimental}

Elevated Temperature Tensile Test. The nominal composition of the $2195 \mathrm{Al}-\mathrm{Cu}-\mathrm{Li}$ alloy used in this study is presented in Table 1. The material was received from Constellium Global Aerospace, France as $7.8 \mathrm{~mm}$ thick sheets.

Table 1. Composition of the Al-Cu-Li alloy (wt/o).

\begin{tabular}{|c|c|c|c|c|c|c|c|c|c|c|c|c|c|}
\hline $\mathbf{A l}$ & $\mathbf{A g}$ & $\mathbf{C r}$ & $\mathbf{C u}$ & $\mathbf{F e}$ & $\mathbf{L i}$ & $\mathbf{M g}$ & $\mathbf{M n}$ & $\mathbf{N i}$ & $\mathbf{P b}$ & $\mathbf{S i}$ & $\mathbf{T i}$ & $\mathbf{Z n}$ & $\mathbf{Z r}$ \\
\hline 94.56 & 0.19 & 0.0004 & 3.76 & 0.05 & 1.00 & 0.28 & 0.02 & 0.0015 & 0.0003 & 0.02 & 0.01 & 0.001 & 0.11 \\
\hline
\end{tabular}

The main purpose of elevated temperature tensile tests was to investigate the superplastic behavior of this alloy. Testing was accomplished at temperatures of $430^{\circ} \mathrm{C}, 460^{\circ} \mathrm{C}$, and $490^{\circ} \mathrm{C}$, and strain rates of $1 \times 10^{-4}, 5 \times 10^{-4}$, and $1 \times 10^{-3} / \mathrm{sec}$. The flow stress behavior was obtained from a series of tensile tests and the strain rate sensitivity, $\mathrm{m}$, was determined from the following:

$$
m=\ln \left(\sigma_{2} / \sigma_{1}\right) \ln \left(\dot{\varepsilon}_{2} / \dot{\varepsilon}_{1}\right),
$$

where, subscripts ' 1 ' and ' 2 ' stand for the different strain rate conditions.

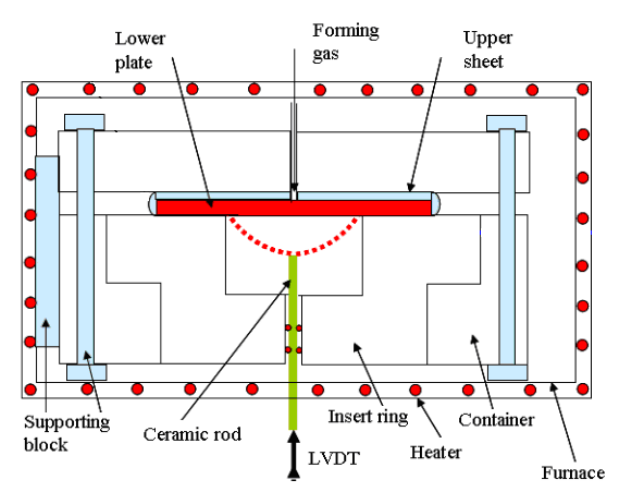

(a)

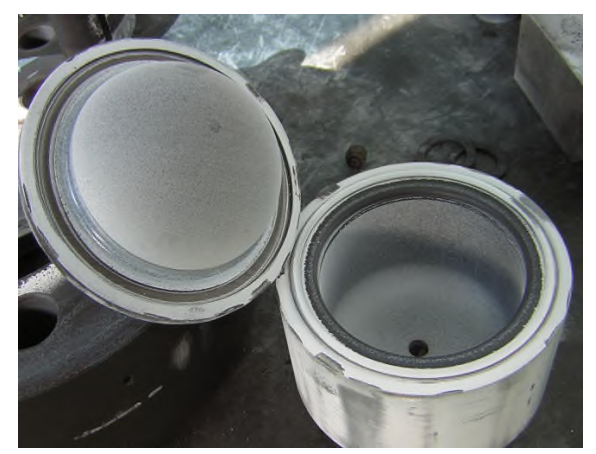

(b)

Fig. 1 (a) Schematic view of the bulging test apparatus [6] and (b) photograph of tool and specimen.

Bulging Test. The biaxial bulging test method was previously described [6-8]. The analyses of the hydrostatic bulging of thin sheets into cylindrical die provided the equivalent relationships between biaxial bulge forming and tensile test. If the height change according to time is measured throughout the experiment, it is possible to obtain the strain rate and flow stress from the analysis. The argon gas was applied inside of the tool for bulge test. The diameter of biaxial test specimen was $100 \mathrm{~mm}$ and the 
height of the free bulged sheet was measured with a high temperature LVDT according to time. The constant hydrostatic pressure was maintained until the height reached to $40 \mathrm{~mm}$ at $1.5 \mathrm{MPa}$ to $2.5 \mathrm{MPa}$. The measured data was transformed into strain rate and stress based on the membrane theory.

\section{Results and Discussion}

Microstructure of the specimen is shown in Fig. 2. The average grain size is about 17 micron. Since there are many different types of precipitates, it is necessary to identify each precipitate with electron microscope as shown in Fig. 2(c). The precipitate \#1 is a GP zone and \#2 is identified as $\theta$ ' or $\theta$ phase located at grain boundaries and aggregated together. The precipitate \#3 is especially important to play a key role for hardening in this alloy. This is high modulus, coherent $\beta^{\prime}\left(\mathrm{Al}_{3} \mathrm{Zr}\right)$ phases, which maintains the microstructure suitable for superplasticity since it is a recrystallization inhibitor at elevated temperature. It inhibit subsequent recrystallization by pinning migrating grain boundaries. Unlike other precipitates, this phase is stable at high temperatures, since the melting temperature of this phase is $1,350{ }^{\circ} \mathrm{C}$. This phase suppress the grain boundary mobility and maintain the grain size constant at superplastic forming temperatures.

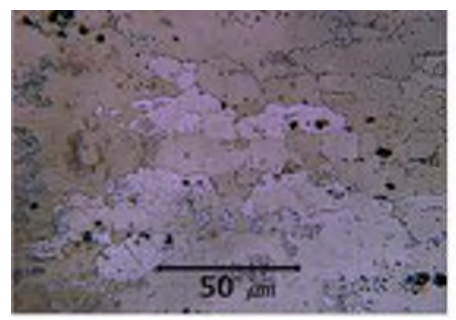

(a)

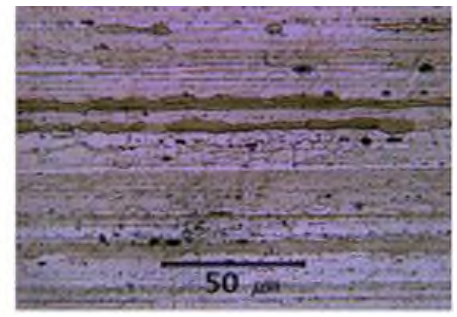

(b)

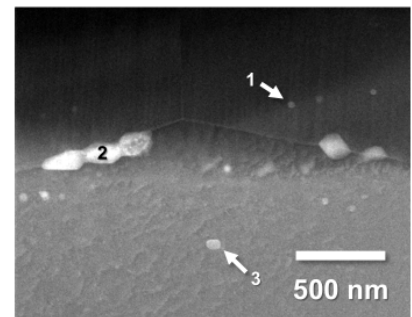

(c)

Fig. 2 Microstructure of (a) longitudinal and (b) transverse view from metallurgical microscope, and

(c) precipitates from transmission electron microscope [9].

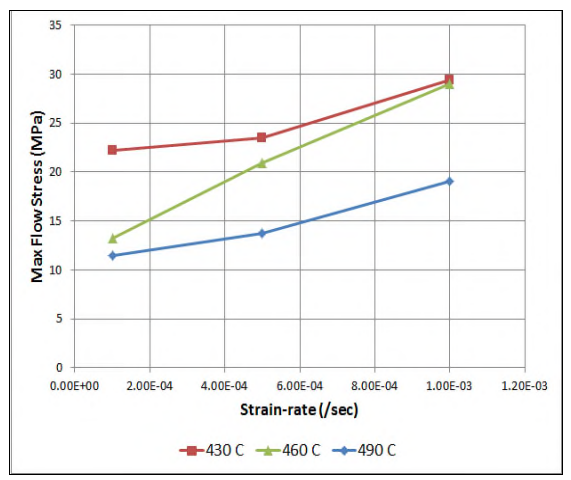

(a)

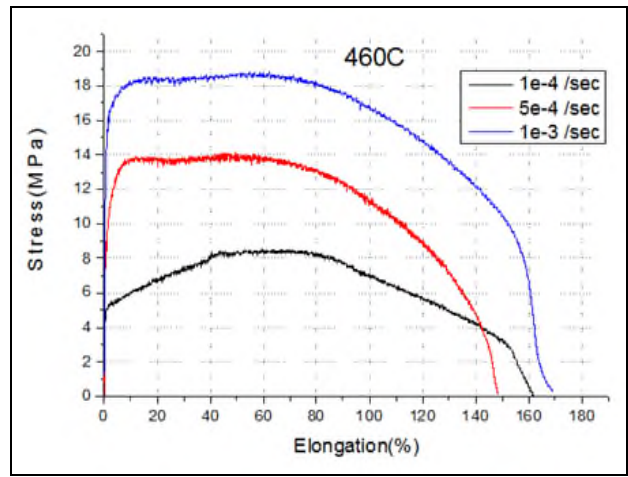

(b)

Fig. 3 (a) Schematic view of the bulging test apparatus and (b) photograph of tool and specimen.

The result of elevated tensile tests is shown in Fig. 3. At elevated temperature, the empirical and experimental relationship between flow stress and strain rate is usually simplified as following:

$$
\sigma=E \dot{\epsilon}^{m}
$$

where, $\sigma$ is the flow stress, E a material strength constant which depends on microstructure, and $\mathrm{m}$ is the strain rate sensitivity. The effect of the rate of deformation on the flow stress is important in elevated temperature deformation. From this information, the strain rate sensitivity and strength coefficient have been calculated from the log-scaled plot of stress vs. strain rate. At $460^{\circ} \mathrm{C}$ the strain rate sensitivity of 0.38 is obtained at the strain rate of $10^{-3} / \mathrm{sec}$.

The result of bulging test is summarized in Table 1 . Based on the optimum superplastic condition from tensile tests, the testing temperatures of $445^{\circ} \mathrm{C}, 460^{\circ} \mathrm{C}$, and $475^{\circ} \mathrm{C}$ are selected. In bulging test, 
the strain rate sensitivity of 0.44 is obtained at $460^{\circ} \mathrm{C}$. At this temperature, with increasing bulging height, the change of the strain rate sensitivity is shown in Fig. 4. The strain rate sensitivity obtained from the biaxial test (0.44) was slightly higher than that from the tensile test $(0.38)$ for this alloy at $460^{\circ} \mathrm{C}$. This is because of different loading conditions between uniaxial tensile and biaxial stress. It is notable that the equivalent stress and equivalent strain rate are incorporated in bulging test. In industrial application of sheet metal forming, the result from bulging test is expected to provide more precise information since it represents the practical loading condition in which the sheet metal undergoes during the forming process.

Table 1. Result of bulging test at each temperatures.

\begin{tabular}{|c|c|c|}
\hline Temperature, ${ }^{\circ} \mathrm{C}$ & $\mathrm{m}$ & Strain rate range, $/ \mathrm{sec}$ \\
\hline 445 & 0.32 & $0.4 \sim 2 \times 10^{-4}$ \\
\hline 460 & 0.44 & $0.65 \sim 4 \times 10^{-4}$ \\
\hline 475 & 0.36 & $2.0 \sim 4 \times 10^{-4}$ \\
\hline
\end{tabular}

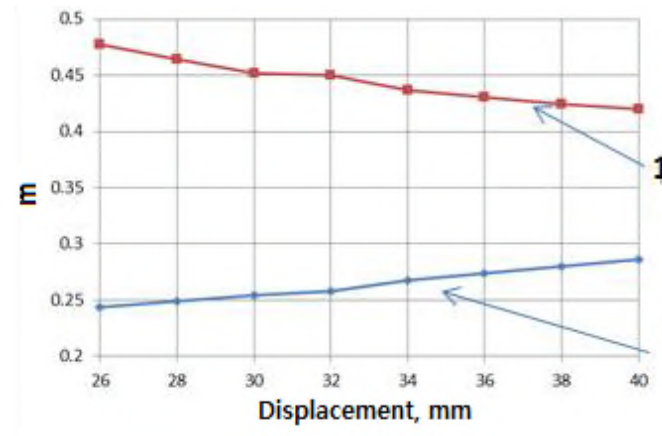

(a)

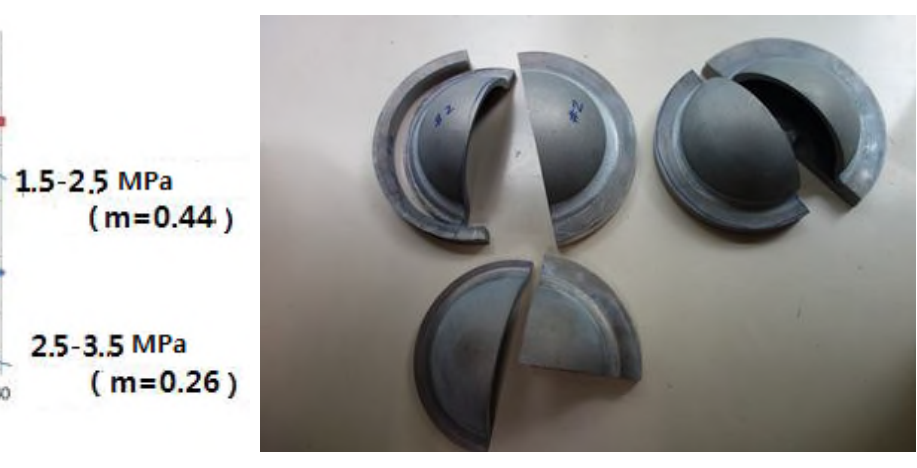

(b)

Fig. 4 (a) Strain rate sensitivity as a function of bulging height, and (b) a photograph of bulged specimens.

\section{Summary}

Aluminum-lithium alloy plays an important role in aerospace industry due to the high specific strength and modulus with good formability. Many aluminum-lithium alloys have been known to have a superplastic property so that using superplastic forming can add additional advantages for these alloy. In this study, superplastic property of 2195 aluminum-lithium alloy is characterized with elevated tensile test and biaxial bulging test. Even though the tensile test is relatively simple, it bears relatively little resemblance to real industrial forming process of sheet metals. In industrial sheet metal forming practice, biaxial stress state is important especially considering plastic anisotropy. In biaxial bulging tests, the strain rate in which the metal sheet experiences varies at constant hydrostatic pressure and the strain rate sensitivity is determined in a single loading of gas pressure. Tensile tests were also performed at elevated temperature and materials parameters were compared with the one obtained from biaxial bulging tests. The strain rate sensitivity obtained from the biaxial test was slightly higher than that from the tensile test for this alloy at $460^{\circ} \mathrm{C}$. This is because equivalent stress and equivalent strain rate are incorporated in bulging test considering anisotropic properties of rolled sheets. The results can be applied directly to the practical superplastic forming process of 2195 aluminum-lithium alloy. 


\section{References}

[1] H. Chokshi, A. K. Mukherjee, T. G. Langdon, Superplasticity in Advanced Materials, Mater. Sci. Eng. Rep. R10 (1993) 237-274.

[2] A. K. Mukherjee, J. E. Bird, J. E. Dorn, Experimental correlation for high-temperature creep, ASM Trans. Q. 62 (1969) 155-215.

[3] J. E. Bird, A. K. Mukherjee, J. E. Dorn, Correlations between High-Temperature Creep Behavior and Structure. In Proceedings of a Symposium on Quantitative Relation Between Properties and Microstructure, Haifa, Israel, 27 July-1 August 1969; Eds. By D. G. Brandon, A. Rosen; Isreal University Press: Jerusalem, Isreal, 1969, pp. 225-342.

[4] https://www.nasa.gov/pdf/63752main_ET_Overview_Wanda_print.pdf, Space Shuttle External Tank Overview.

[5] K. K. Sankaran, J. E. O'Neal, Structure-property relationships in Al-Cu-Li alloys. In Aluminium-Lithium alloys, Eds. by T. H. Sanders, E. A. Starke; Metall. Soc. AIME: Warrendale, PA, 2 (1984) 393-405.

[6] H. S. Lee, J. H. Yoon, J. T. Yoo, An experimental study on elevated temperature biaxial bulge test, Adv. Mater. Res. 430-432 (2012) 539-542.

[7] A. Dutta, A. K. Mukherjee, Superplastic forming: an analytical approach, Mater. Sci. Eng. A 157 (1992) 9 .

[8] G. Giuliano, S. Franchitti, The determination of material parameters from sperplastic free-bulging tests at constant pressure, Int. J. Mach. Tools Manuf. 48 (2008) 1519-1522.

[9] J. H. Kim, et al., Effect of precipitates on mechanical properties of AA2195, J. Alloy. Comp. 669 (2016) 187-198. 